\title{
Fuzzy Weighted Least Square Filter for Pansharpening in Satellite Images
}

\author{
Jegatheeswari P \\ Department of VLSI design, \\ Dr. Sivanthi Adithanar college of Engineering \\ Tuticorin, Tamil Nadu, India \\ jegatheeswari11@gmail.com
}

\author{
Angelin Deepa T \\ Department of Computer Science and Engineering, \\ Bethlahem College of Engineering \\ Ulaganvillai, Tamil Nadu, India \\ Deepalibin1992@gmail.com
}

\begin{abstract}
Many remote sensing applications require images with both high spatial and spectral resolution. But due to the technological limitations of satellite sensors, the spatial and spectral details will not present in a single image. Pansharpening combines a low spatial resolution multispectral image with a high spatial resolution panchromatic image to create a fused color image with both spatial and spectral resolution. There are a number of applications in remote sensing that require images with both high spatial and spectral resolutions. Accordingly, a technique is proposed for pansharpening using Fuzzy weighted least square filter that intends to eliminate the low frequency components of the pan and MS images. The proposed FWLS filter estimates the low frequency components of the pan and MS images before finding the detail map. Then, the spectral signatures of the different bands of the MS image are refined with the detail map estimated. Finally, the refined detail maps are injected into the corresponding bands of the MS image so as to generate the high resolution multispectral image. The performance of the proposed method is evaluated using six metrics, such as CC, ERGAS, UIQI, SAM, RASE, and RMSE, respectively
\end{abstract}

Keywords: Remote sensing, satellite image, Pan-sharpening, Fuzzy weighted least square filter, high resolution image.

\section{Introduction}

Satellites provide very valuable information, making it suitable for various applications, like weather prediction, environmental monitoring, military intelligence, and so on. As satellites are costly, it is necessary to utilize the complete information attained from the satellites available, e.g., by incorporating the output from several sensors. The remote sensing satellite sensors have several limitations, such as less space for data storage, minimum bandwidth for communication with earth station etc. So, the remote sensing satellites cannot capture the high spatial and spectral resolution images simultaneously. Generally, the satellite sensors capture the remote sensing images as two types of images such as a panchromatic image and a multispectral image. The panchromatic image gives clear information about the spatial resolution, while the color (spectral) information will be missing. In converse, the multispectral image provides clear information about the color, but the spatial information will be missing [1]. One of the best examples is merging of satellite images of low spatial and high spectral resolution with images of high spatial and low spectral resolution. This process is known as image fusion or pansharpening. Pansharpening can be a key preprocessing step in remote sensing.

Pan sharpening is a technique for fusing the panchromatic image and multispectral images, which combines the high resolution panchromatic image and low resolution multispectral image to generate an image with both high spatial and spectral resolution. Various pansharpening techniques have been developed in the recent years. Most of those techniques have to compromise between the desired spatial enhancement and the spectral consistency. Attaining better spectral consistency usually compromises the spatial resolution and vice versa. Pansharpened images are commonly not used directly for classification, rather, pansharpening is used to improve the image visually.

In this paper, pansharpening with the aid of Fuzzy weighted least square filter, which eliminates the low frequency components of the pan and MS images, is proposed. Here, the low frequency components of the pan and MS images are computed using the proposed FWLS filter before finding the detail map. Moreover, the detail map estimated is refined with the spectral signatures of the different bands of the 
MS image. Once the spectral signatures are refined with the detail map, the refined detail maps are injected into the corresponding bands of the MS image to obtain the high resolution multispectral image.

The organization of the paper is as follows: Section 2 briefly describes some of the existing filtering based pansharpening techniques. The proposed work is described in section 3 . Section 4 provides the results and discussion section and the conclusion is provided in section 5 .

\section{Literature Survey}

Among the various pan sharpening techniques, the most commonly used techniques are intensity-huesaturation (IHS) method [2], Principal Component Analysis (PCA) [3] and Brovey Transform (BT) [4]. These methods can maintain the spatial resolution same as the panchromatic image but the spectral characteristics of the multispectral image will be distorted. Wavelet transform based technique preserves the spectral information of the image but it does not preserve the spatial information[5].

Pan sharpening methods based on filters are also common, in which the spatial information is extracted from the panchromatic image and it is injected into the multispectral image. In High Pass Filtering (HPF) method [6], high pass filter is used to extract the high frequency components of the panchromatic image and this high frequency component is added to each band of the multispectral image. This method preserves the spectral characteristics of the multispectral image. In [7], Kaplan and Erer proposed a technique in which a multiscale bilateral filter is used to decompose the high spatial resolution pan image and it is used as the high frequency information of the low resolution multispectral image. Pan sharpening based on subband decomposition of pan and multispectral images using 1-D lattice filter structure is proposed in [8]. In this approach, the pansharpened image is obtained by combining the sub band of bothimages using undecimated lattice reconstruction structure and some predefined rules. An adaptive pansharpening technique using weighted least square (WLS) filter is proposed in [9]. This approach uses WLS filter to eradicate the influence of the low-frequency components of the Pan and MS images. The experimental result shows that this approach performs well in preserving both spatial and spectral characteristics.

\section{Proposed Work}

IHS method is a very basic and simple technique in pan sharpening of satellite images [2]. But this method has several drawbacks such as the presence of low frequency components in the detail map results in spectral distortion and local artifacts may arise because all the bands of the multispectral image share the same detail map. In order to solve these problems, FuzzyWeighted least square filter is proposed in this work, which eliminates the influence of low frequency components. The FWLS filter estimates the low frequency components of the pan and MS images before finding the detail map. Furthermore the estimated detail map is refined with the spectral signatures of the different bands of the MS image. Then the refined detail maps are injected into the corresponding bands of the MS image to obtain the high resolution multispectral image.

The FWLS filter is an edge preserving filter, used to smooth the images without affecting the edges of the image. The edges are preserved by compromising between sharpening and blurring. In this work, the FWLS filter is used as low pass filter to estimate the low frequency components of the pan and MS images.

For an input image $I$, we require a filtered image $I_{f}$, which should be maximally close to $I$ and as smooth as possible everywhere, except the edges. The filtered image $\mathrm{I}_{\mathrm{f}}$ can be obtained as follows:

$$
\arg \min _{I_{f}}\left(\left\|I_{f}-I\right\|^{2}+\lambda\left(w_{x}\left(\frac{\partial I_{f}}{\partial x}\right)^{2}+w_{y}\left(\frac{\partial I_{f}}{\partial y}\right)^{2}\right)\right)
$$

where, the first term $\left\|I_{\mathrm{f}}-\mathrm{I}\right\|^{2}$ ensures that the distance between $\mathrm{I}_{\mathrm{f}}$ and Iis minimum. The second term is for achieving smoothness by minimizing the partial derivative of $\mathrm{I}_{\mathrm{f}} \cdot w_{x}$ and $w_{y}$ are smoothness weights. $\lambda$ is the regularization factor for balancing the two terms.

The flowchart of the proposed method is given in figure 1. 


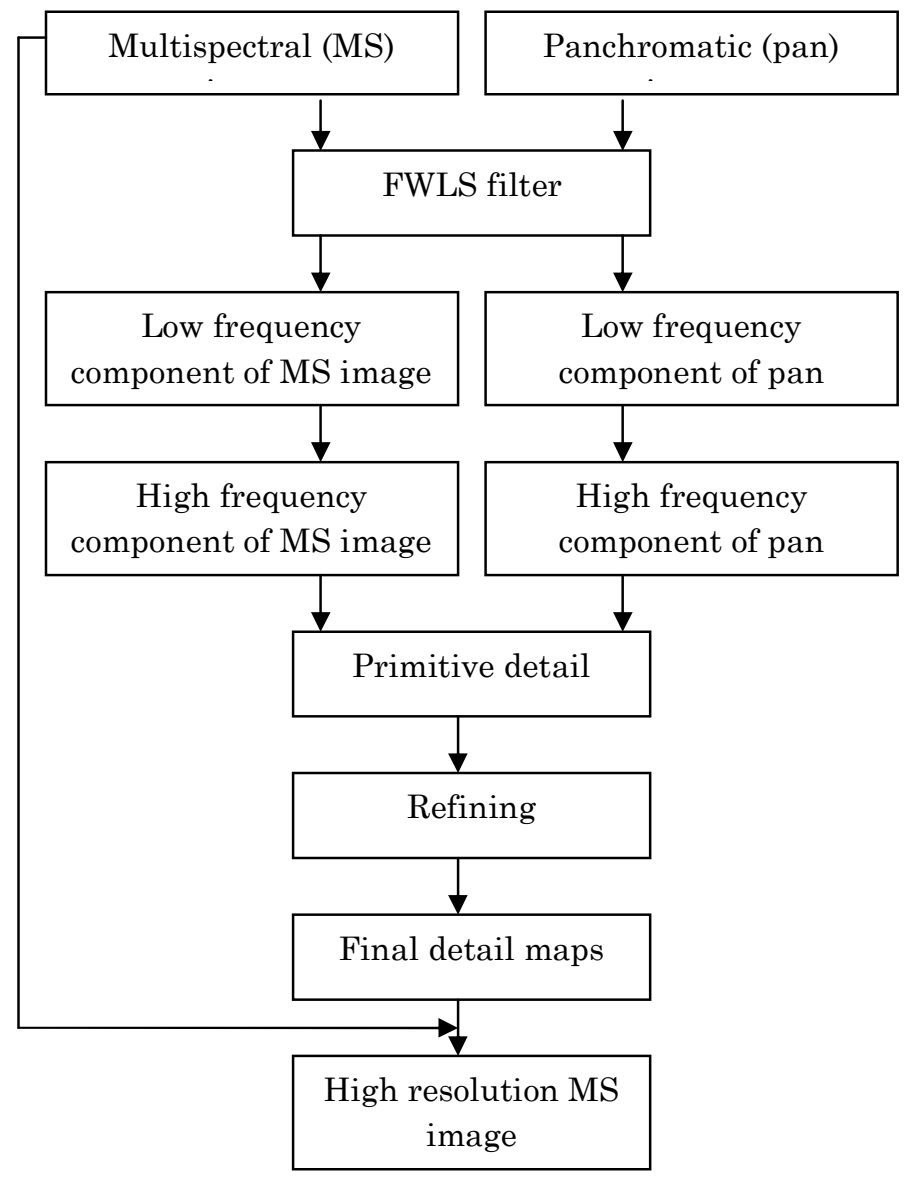

Figure 1. Flow chart of the proposed method

Initially, the low frequency component of the MS image $\left(\mathrm{MS}_{\mathrm{L}}\right)$ and the pan image $\left(\mathrm{P}_{\mathrm{L}}\right)$ is estimated using the FWLS filter. The difference between the original images and the low frequency components are considered as the high frequency components $\left(\mathrm{MS}_{\mathrm{H}}\right.$ and $\left.\mathrm{P}_{\mathrm{H}}\right)$. Based on the high frequency components, the primitive detail map is estimated. The estimated primitive detail map may not be optimal, because different bands of the high resolution multispectral image share the same detail map, which may cause local artifacts. So, the primitive detail map is refined to generate final detail maps for each band. Finally, the primitive detail map is injected into the corresponding bands of the multispectral image to obtain the high resolution multispectral image.

\subsection{Estimation of Primitive Detail Map}

The decomposition of the pan and MS images into low frequency and high frequency components are expressed as follows.

$$
\begin{aligned}
& \mathrm{P}=\mathrm{P}_{\mathrm{L}} \oplus \mathrm{P}_{\mathrm{H}} \\
& \mathrm{MS}^{\mathrm{i}}=\mathrm{MS}_{\mathrm{L}}^{\mathrm{i}} \oplus \mathrm{MS}_{\mathrm{H}}^{\mathrm{i}}
\end{aligned}
$$

where, $\oplus$ refers to an operation which combines the low frequency components and high frequency components into an image. $\mathrm{P}_{\mathrm{L}}$ and $\mathrm{P}_{\mathrm{H}}$ are the low frequency components and high frequency components of the pan image, respectively. $\mathrm{MS}^{\mathrm{i}}$ represents the $i^{\text {th }}$ band if the MS image. $\mathrm{MS}_{\mathrm{L}}^{\mathrm{i}}$ and $\mathrm{MS}_{\mathrm{H}}^{\mathrm{i}}$ are the low frequency components and high frequency components of the MS image, respectively.

In order to eliminate the influence of low frequency components, the primitive detail map $D$ is estimated as follows:

$$
\mathrm{D}=\mathrm{P}_{\mathrm{H}} \quad \mathrm{I}_{\mathrm{H}}
$$

where, $\mathrm{I}_{\mathrm{H}}$ represents the high frequency components of the intensity band $I$ and it is calculated as follows: 


$$
\mathrm{I}_{\mathrm{H}}=\sum_{\mathrm{i}=1}^{\mathrm{N}} \alpha^{\mathrm{i}} \mathrm{MS}_{\mathrm{H}}^{\mathrm{i}}
$$

where, $\alpha^{\mathrm{i}}$ is the band coefficient of $M S_{H}^{i}$.

From equ. (3) and (4), it is clear that, to estimate the primitive detail map D, we need to calculate $\mathrm{P}_{\mathrm{H}}, \alpha^{\mathrm{i}}$ and $\mathrm{MS}_{\mathrm{H}}^{\mathrm{i}}$.

The low frequency components $P_{L}$ and $M S_{L}^{i}$ are obtained by applying the FWLS filter.

$$
\begin{aligned}
& P_{L}=F(P) \\
& \mathrm{MS}_{\mathrm{L}}^{\mathrm{i}}=\mathrm{F}\left(\mathrm{MS}^{\mathrm{i}}\right)
\end{aligned}
$$

where, $F(\bullet)$ refers to the operator of FWLS filter. By applying inverse operation on equ. (1) and (2), $P_{H}$ and $M S_{H}^{i}$ can be obtained, respectively.

Using $\mathrm{I}_{\mathrm{H}}, \mathrm{P}_{\mathrm{H}}$ can be approximated as close as possible [10]. Therefore,

$$
\mathrm{P}_{\mathrm{H}} \approx \sum_{\mathrm{i}=1}^{\mathrm{N}} \alpha^{\mathrm{i}} \mathrm{MS}_{\mathrm{H}}^{\mathrm{i}}
$$

Using least square estimation, the optimal coefficient $a^{i}$ is calculated as follows:

$$
\alpha^{\mathrm{i}}=\left(\left(\mathrm{MS}_{\mathrm{H}}^{\mathrm{i}}\right)^{\mathrm{T}} \mathrm{MS}_{\mathrm{H}}^{\mathrm{i}}\right)^{1}\left(\mathrm{MS}_{\mathrm{H}}^{\mathrm{i}}\right)^{\mathrm{T}} \mathrm{P}_{\mathrm{H}}
$$

Using $\alpha^{\mathrm{i}}, \mathrm{I}_{\mathrm{H}}$ can be obtained as shown in eqn. (4), and from eqn. (3), the primitive detail map can be obtained.

\subsection{Refinement of Primitive Detail Map}

In general, after obtaining the detail map, the high resolution multispectral (HMS) image is formed as follow.

$$
\mathrm{HMS}^{\mathrm{i}}=\mathrm{MS}^{\mathrm{i}}+\mathrm{D}
$$

But, here it is considered that, each band of the MS each has its own detail map. Therefore, the above equation can be rewritten as,

$$
\mathrm{HMS}^{\mathrm{i}}=\mathrm{MS}^{\mathrm{i}}+\mathrm{D}^{\mathrm{i}}
$$

where, $\mathrm{D}^{\mathrm{i}}$ indicated the final detail map of the $i^{\text {th }}$ band.

It is assumed that the low frequency component of the MS image are approximately equal to the low frequency components of the HMS image. After neglecting the low frequency component in eqn. (10), we have,

$$
\mathrm{HMS}_{\mathrm{H}}^{\mathrm{i}}=\mathrm{MS}_{\mathrm{H}}^{\mathrm{i}}+\mathrm{D}^{\mathrm{i}}
$$

where, $\mathrm{HMS}_{\mathrm{H}}^{\mathrm{i}}$ indicates the high frequency component of the ith band of the HMS image. Similar to eqn. (4), high frequency component of the intensity band of $\mathrm{HMS}$ image $\mathrm{HI}_{\mathrm{H}}$ can be represented as,

$$
\mathrm{HI}_{\mathrm{H}}=\underset{\mathrm{i}=1}{\mathrm{~N}} \alpha^{\mathrm{i}} \mathrm{HMS}_{\mathrm{H}}^{\mathrm{i}}=\underset{\mathrm{i}=1}{\mathrm{~N}} \alpha^{\mathrm{i}}\left(\mathrm{MS}_{\mathrm{H}}^{\mathrm{i}}+\mathrm{D}^{\mathrm{i}}\right)
$$

For each pixel, if $\mathrm{HI}_{\mathrm{H}}$ is closer to $\mathrm{P}_{\mathrm{H}}$, then the spatial quality of the fused high resolution multispectral image will be higher. With respect to the estimated final detail map, the function $F$ is minimized as follows:

$$
\arg \min _{D^{i}} F\left(D^{i}\right)=\left\|P_{H} \sum_{i=1}^{N} \alpha^{i}\left(M_{H}^{i}+D^{i}\right)\right\|^{2}
$$

By refining the primitive detail map $\mathrm{D}, \mathrm{D}^{\mathrm{i}}$ can be calculated with the steepest descent method. Further updation of $\mathrm{D}_{\mathrm{i}}$ can be done as follows:

$$
\mathrm{D}_{(\mathrm{n}+1)}^{\mathrm{i}}=\mathrm{D}_{(\mathrm{n})}^{\mathrm{i}} \quad \gamma \frac{\partial \mathrm{F}}{\partial \mathrm{D}^{\mathrm{i}}} \quad \mathrm{D}^{\mathrm{i}}=\mathrm{D}_{(\mathrm{n})}^{\mathrm{i}}
$$


where, the initial value $D_{(0)}^{i}=D$, and $\gamma$ is the step size and it must be a small positive number. The iterative process will converge to a solution. After finding the final detail map $\mathrm{D}^{\mathrm{i}}$, the high resolution multispectral image can be obtained by using eqn. (10).

\section{Experimental Results and Analysis}

\subsection{Experimental Setup}

The input pan image, low resolution multispectral image and the output high resolution multispectral image are shown in figure 2.

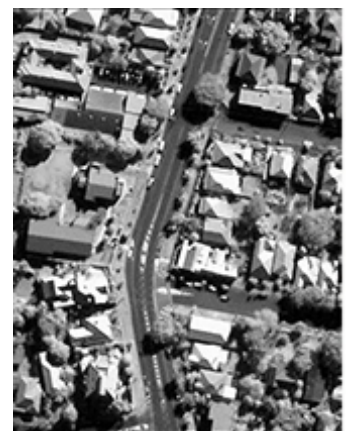

a)

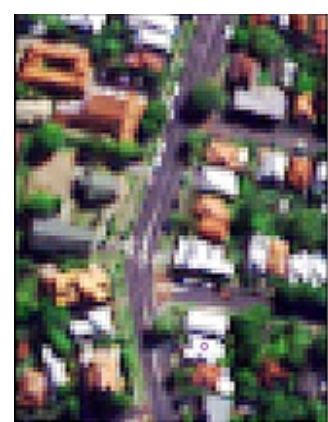

b)

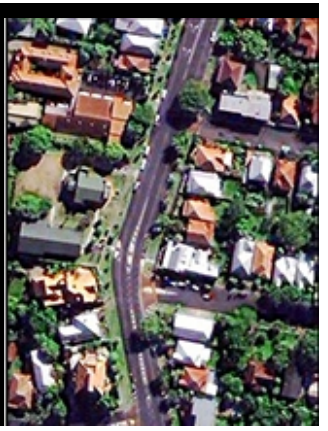

c)

Figure 2. Input and output images a) pan image b) low resolution MS image

c) high resolution $M S$ image

The multispectral images of these satellites contain four bands such as R,G,B and NIR. Initially, the original pan images and multispectral images are downsampled to a lower resolution by a downsampling factor of $1 / 4$. The downsampled multispectral image is sharpened back to its original resolution, and the original multispectral images are regarded as the high resolution multispectral image for reference. The parameters are set as follows: The regularization factor $\lambda=0.8$ and the step size $\gamma=0.1$. Sixpansharpening methods such as IHS method [9],AIHS method [11], the À trous wavelet transformbased (AWLP) method [12], the Generalized Laplacian Pyramid (GLP) method [13], the Gram-Schmidt Adaptive (GSA) method [14], and the Bilateral filter-based (BFLP) method [15] are used for the comparing the performance of the proposed method.

Six global metrics are utilized to evaluate the performance of different pansharpening methods.

i) Correlation coefficient (CC)

It measures the correlation between the sharpened result and the reference. The sharpened result is closer to the reference when CC is smaller.

ii) Relative dimensionless global error in synthesis (ERGAS)

It measures the global quality of the sharpened result. The better sharpened result has a smaller value.

iii) Universal image quality indexes (UIQI)

It reflect the loss of correlation, luminance distortion, and contrast distortion. The higher the value of UIQI, the higher the spectral quality. The highest value for UIQI is 1.

iv) Spectral angle mapper (SAM)

It denotes the absolute value of the spectral angle between the sharpened result and the reference vectors. A small SAM value means small spectral angle distortion.

v) Relative average spectral error (RASE)

It characterizes the average performance in the spectral bands. The lower the value of RASE, the higher the spectral quality.

vi) Root-mean-square error (RMSE)

It indicates the average esquared difference between the sharpened result and the reference. The smaller the RMSE, the better the performance is.

\subsection{Comparison of different pansharpening methods:}

The quantitative evaluation of the different pansharpening methods are given in table 1. From the table it is clear that the proposed method outperforms other methods interms of both spatial and spectral 
quality. For the comparison, it can be noted that the high resolution image obtained by the proposed method is similar to the reference high resolution multispectral image.

Table 1. Performance evaluation

\begin{tabular}{llll|ll|l|}
\hline & CC & ERGAS & UIQI & SAM & RASE \\
\hline IHS & 0.942 & 6.324 & 0.869 & 0.071 & 23.675 & 74.986 \\
AIHS & 0.933 & 6.107 & 0.932 & 0.063 & 23.882 & 73.494 \\
AWLP & 0.946 & 5.994 & 0.941 & 0.059 & 24.092 & 74.142 \\
GLP & 0.952 & 5.487 & 0.950 & 0.065 & 21.865 & 24.100 \\
GSA & 0.944 & 6.207 & 0.940 & 0.074 & 20.061 \\
BFLP & 0.959 & 4.941 & 0.957 & 0.056 & 74.167 \\
Proposed & 0.967 & 3.678 & 0.969 & 0.047 & 51.735 \\
\hline
\end{tabular}

\section{Conclusion}

In this paper, a novel pansharpening method using Fuzzy Weighted Least Square filter was proposed. Different from the existing methods, the proposed method used FWLS filter to eliminate the low frequency components of the pan image and multispectral image. The proposed FWLS filter is used to calculate the low frequency components of the pan and MS images before finding the detail map. Furthermore, the spectral signatures of the different bands of the MS image are refined with the detail map. Then, the refined detail maps are injected into the corresponding bands of the MS image so as to generate the high resolution multispectral image. The performance of the proposed method is evaluated using CC, ERGAS, UIQI, SAM, RASE, and RMSE, respectively. On comparing with the existing methods, the proposed method performs well in terms of both spatial and spectral quality.

\section{Compliance with Ethical Standards}

Conflicts of interest: Authors declared that they have no conflict of interest.

Human participants: The conducted research follows the ethical standards and the authors ensured that they have not conducted any studies with human participants or animals.

\section{References}

[1] C. Pohl, J. L. Van Genderen, Multisensor image fusion in remote sensing: Concepts, methods and applications, Int. J. Remote Sens. 19, (1998) 823-854.

[2] T. Tu, S. Su, H.Shyu, P. Huang, A new look at IHS-like image fusion methods, Inf. Fusion, 2, (2001) $177-186$.

[3] V.P Shah, N.H. Younan, An efficient pan-sharpening method via a combined adaptive PCA approach and contourlets, IEEE Trans. Goesci. Remote Sens., 46, (2008)

[4] Yuhong Ding and Yanhui Wang, "Analysis and Evaluation on Fusion Methods of Medium and High Spatial Resolution Remote Sensing Image”, IEEE, 19th International Conference on Geoinformatics, 2011, pp. $1-4$

[5] K. Amolins, Y. Zhang, P. Dare, Wavelet based image fusion techniques-An introduction, review and comparison, ISPRS J. Photogramm, Remote Sens., 62, (2007) 249-263.

[6] Chavez, P., C.Sides, and J. Anderson. 1991. "Comparison of Three different methods to merge multiresolution and multispectral data: Landsat Tm and Spot Panchromatic. "Photogrammetric Engineering and remote sensing 57: 295-303

[7] N.H. Kaplan, I. Erer, "Bilateral filtering-based enhanced pansharpening of multispectral satellite images", IEEE Geoscience and Remote Sensing Letters, vol. 11, no. 11, pp. 1941-1945, 2014

[8] N.H. Kaplan, I. Erer, "Pansharpening of Multispectral Satellite Images via Lattice Structures". International Journal of Computer Applications 140(7):9-14, April 2016.

[10] S. Rahmani, M. Strait, D. Merkurjev, M. Moeller, and T. Wittman, "An adaptive IHS pan-sharpening method," IEEE Geosci. Remote Sens. Lett., vol. 7, no. 4, pp. 746-750, Oct. 2010.

[11] S. Rahmani, M. Strait, D. Merkurjev, M. Moeller, and T. Wittman,"An adaptive IHS pan-sharpening method," IEEE Geosci. Remote Sens.Lett., vol. 7, no. 4, pp. 746-750, Oct. 2010.

[12] X. Otazu, M. Gonzalez-Ausicana, O. Fors, and J. Nunez, "Introductionof sensor spectral response into image fusion methods: Application towavelet-based methods," IEEE Trans. Geosci. Remote Sens., vol. 43,no. 10, pp. 2376-2385, Oct. 2005.

[13] B. Aiazzi, L. Alparone, S. Baronti, A. Garzelli, and M. Selva, "MTFtailored multiscale fusion of high-resolution MS and Pan imagery,"Photogramm. Eng. Remote Sens., vol. 72, no. 5, pp. 591-596, May 2006.

[14] B. Aiazzi, S. Baronti, and M. Selva, "Improving component substitution pansharpening through multivariate regression of MS+Pan data," IEEE Trans. Geosci. Remote Sens., vol. 45, no. 10, pp. 3230-3239, Oct. 2007.

[15] N. H. Kaplan and I. Erer, "Bilateral filtering-based enhanced pansharpening of multispectral satellite images," IEEE Geosci. Remote Sens. Lett., vol. 11, no. 11, pp. 1941-1945, Nov. 2014. 